\title{
Performance Gain in HIVE through Query Optimization using Index Joins
}

\author{
Stephen Neal Joshua Eali ${ }^{1}$, Nakka Thirupathi Rao ${ }^{1}$, Swathi Kalam ${ }^{1}$, \\ Debnath Bhattacharyya ${ }^{1}$ and Hye-jin Kim ${ }^{2}$ \\ Department of Computer Science \& Engineering, Vignan's Institute of \\ Information Technology, Visakhapatnam-530049, India \\ ${ }^{2}$ Business Administration Research Institute, Sungshin W. University, \\ 2, Bomun-ro 34da-gil, Seongbuk-gu, Seoul \\ nakkathiru@gmail.com,debnathb@gmail.com, \\ hyejinaa@daum.net (Corresponding Author)
}

\begin{abstract}
Index joins range unit pivotal for proficiency and quality once technique questions over colossal data. HIVE may be a cluster balanced immense data administration motor that is good for data examination applications and for OLAP for phenomenally "specific" inquiries whose yield sizes region unit little division from the contributing data, there the beast compel experiences poor execution because of repetitive circle I/O operations or end in starts of additional guide operations. Here all through this paper a shot is made and propose file joins procedure to rush up the inquiry strategy and incorporate it in Hive by mapping our vogue to the unique change stream to assess the execution, we've a slant to give and measure check inquiries on datasets created abuse TPC-H benchmark. Our outcomes show vital execution increase over moderately tremendous data sets and/or uncommonly specific questions having a two-way are a piece of and one be a piece of condition.
\end{abstract}

Keywords: Hive, Hadoop, Join Operation, Indexing methods, Map and Reduce functions

\section{Introduction}

The launch of net 2.0, parts of the clients and net applications experienced an upset. The inactive view-just clients turned into the substance makers. The likelihood to act over the net allowed to clients, drop all the data from web-based social networking, web journals, recordings and diverse net.2.0 advances to sites has made upgraded hundreds the effectively gathered enormous heap of information on servers. This change requests imaginative answers for store this Brobdingnagian amount of learning and bolster conservative questioning over it. The information must be questioned to separate the commendable data from it. This opens new skylines for improvement of novel calculations, instruments, and administrations to technique inquiries on the available were amount of learning in an exceedingly moderate time span. Hive [7] could be an information stockroom bundle best fitted to OLAP workloads to deal with and question over Brobdingnagian volume of learning living in an exceedingly conveyed capacity. The HDFS is that the framework amid which Hive keeps up the information dependableness and makes due from equipment disappointments. Hive is that the exclusively SQL-like relative colossal data stockpiling approach created on high of Hadoop to the best of our information. As joins are expensive operations in databases that depend on the predicate, information, and so on allowing information "Consolidated" from various relations. It

Received (May 21, 2017), Review Result (August 15, 2017), Accepted (September 11, 2017) 
furthermore gives a considerable measure of data examination and mining errands that are essential inside the setting of business insight for finding captivating and accommodating examples in incredible arrangement of learning. Along these lines, up various be a piece of operations may bring about essential execution change. In relative databases, efficient be a piece of operations are bolstered through order or outer kind procedures, while not that the savage power output of the entire table is miserable for goliath data. The current thing can be considered as a frequently considerable measure of essential particularly once a little portion of the tuples partake in an extremely be a piece of operation.

This paper displays A way to deal with perform be a piece of with MapReduce sort operations, over gigantic arrangements of learning hang on in an extremely Hadoop-based cloud. Assessing by experimentation the execution of the arranged approach, this uses a current order highlight in Hive to upgrade execution over non-listed questions. The remaining set of the paper is sorted out as takes after. Segment II depicts Hive outline and III Section surveys associated work. The considered models inquiry change misuses Index joins are represented in Section IV, and its exploratory investigation and the outcomes are depict in Section V. shutting comments and also the prospect future work are specified in Section VI.

\section{Architecture of the Considered Model Hive}

The proposed hive framework configuration comprises of many parts and their communications and in this manner the Hadoop Map-diminish system. The abnormal state read of this information stockroom configuration is depict in Figure one taken from[7]. At absolute base of Figure one, the Hadoop framework can be observed clearly and in detail for the any sort of users. At the most astounding of Figure one, the raised a piece of Hive is set in partner with its basic segments. A short depiction of those segments and their parts territory unit as takes after:

Meta-store: The currently considered framework of the Hive inventory contains diagrams, tables, segments, and their assortments, tables' areas, measurements and option information basic for learning administration. For instance, in our file joins execution we'd jump at the chance to get a handle on regardless of whether a table said inside the inquiry is filed or not. Or, then again we'd jump at the chance to get a handle on if the list covers every one of the segments of a table. That sort of data is keep inside the meta-store and is asked for by the inquiry compiler simply the once, however the essential a piece of data is transported to many undertaking people who are trackers.

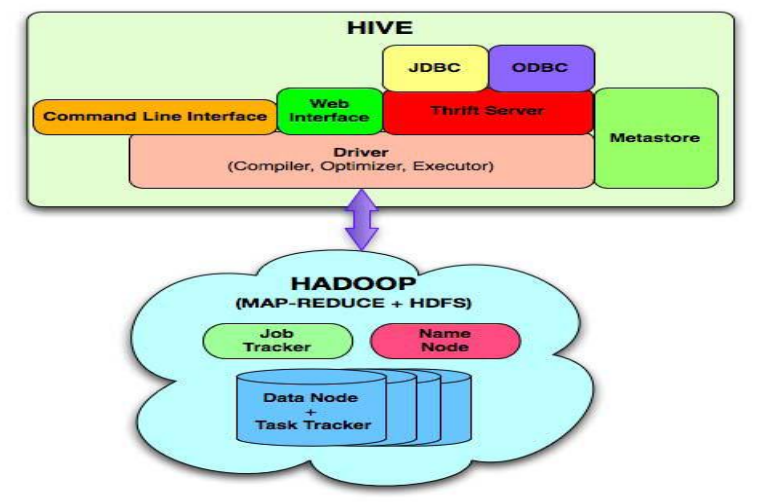

Figure 1. Hive System Architecture

Driver: The part that gets the inquiry, when it's gotten by the user interface as of the client also deals with the era of an inquiry inside Hive. It also actualizes the idea of assembly handles and recovers the session insights. In Figure one, the thought process 
compel comprises of 3 principle parts, specifically, Compiler, Optimizer, and guardian. The analyzer lives at some reason between the compiler and guardian to help the execution. Our anticipated list is a piece of s algorithmic run joins to the compiler and streamlining agent models, which can be said and explained in detail in the coming near future.

\section{Related Work}

Before bouncing to the associated work, it's basic to state the bland change stream in Hive, as most advancement adjusts thereto. The DAG of administrators is passed to the streamlining agent to settle on the best potential grouping of operations on the specific data inside the inquiry organize. Most RDBMSs nowadays get delight from a cost-based inquiry streamlining agent. The mastermind conjointly conveys the required examples or segments if indicated all by itself by the inquiry itself. Hive change incorporates a grouping of changes amid which the administrator DAG yield of 1 change step is sustained as Associate in nursing contribution to ensuing. The place to start to change the streamlining agent or include new change algorithmic program is that the revise interface. To do as such, one should execute the adjust interface abuse their custom rationale to include it to the chain of improvements in Hive Optimizer. Hive streamlining agent will nothing however conjuring all the change, one once another, to change the inquiry orchestrate. The place to start to change the analyzer or include new change algorithmic program is that the revise interface. Hive analyzer will summon all the change, one once another, to change the inquiry organizes.

The following is that the portrayal of its modules and their parts [7].

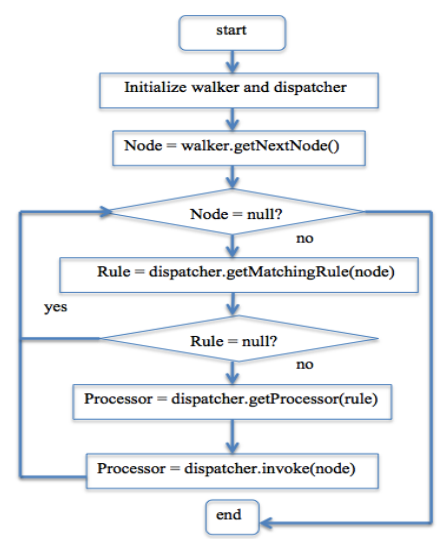

Figure 2. Hive Optimization Flow

\section{i. Generating a query: Using WHERE Clause}

As said in the Hive design, the streamlining agent gets an administrator DAG and plays out the empowered or conceivable advancements. This implies streamlining is connected toward the finish of or amid the legitimate arrangement era organizes. The present case to the intended model is somewhat extraordinary. As a physical advancement it happens all the more unequivocally after the consistent arrangement procedure as the total administrator tree is being exchanged to the tree of errands, yet the present considered model streamlining agent and substantial enhancer contain similar segments we as of now examined and thus the tree experiences comparable strides. The physical arrangement enhancer conjures all the physical advancements thusly. 


\section{ii. Generating a second query: Using GROUP BY Clause}

The objective of the present model and the present case is to quicken inquiries containing GROUP BY conditions. As in HIVE-1644, it utilizes inquiry re-composing strategy, yet its centre plan is not constrained to re-composing as it were. Despite the fact that this streamlining appears to be naturally as material, and within the code it is not composed in the physical advancement bundle, and thus its analyser executes the Transform interface. In its streamlining agent known as RewriteGBUsingIndex.java, it initially verifies the if the inquiry reaches every one requirements, for example,

- The nearness of the file above the join key

- justification of the list

- Reporting of the list above allotments (assuming any)

- Presence of just a single table in the inquiry

- Presence of solitary COUNT (indent_key) work on the inquiry

- Making scarcely the segments that are in the file scratch

\section{iii. Using Indexing Over Mapreduce}

Hive solidifies every important office necessary to execute questions under mapreduce. This implies one can concern an inquiry with no Hive by composing their own guide and diminish techniques and dealing with the question lifecycle themselves. A current work coordinated the record into mapreduce structure, which tries to diminish the quantity of maps created to get to the underlying information utilizing a list with irregular access. The file structure is a B+-tree, where it is not manufactured utilizing a customary make byembed in a best down manner. Rather, since the information and in like manner the record shouldn't be refreshed, the information is perused in bunch mode utilizing the mapreduce system itself; a short time later it is arranged on the (index_key, counterbalanced) combines and composed successively to a document. These sets shape the leaf hubs of the record tree. In the subsequent stage, all the leaf hubs are filtered and the transitional file hubs are made in a base up way.

\section{iv. Query Optimization using Statistics}

Insights assume a key part with regards to inquiry enhancement. Measurements either encourage the analyser to pick the more sparing arrangement, for example, join reordering or fill in as an inquiry yield like the $\operatorname{COUNT}\left({ }^{*}\right)$ condition in a question on their own. Hive gives table and segment level measurements and additionally segment level insights. A current work anticipated putting away segment in Hive tables to gain from all through inquiry execution. Segment level insights or a ton of particularly, histograms that show value dissemination among a table offer a great deal of right data required to evaluate the yield measure. A substitution table is esteem additional to hive meta-store which contains the amount of unmistakable esteems, scope of invalid esteems, min and GHB esteems which are mostly incessant esteems as its fields.

\section{Proposed Index Joins}

The current files in Hive square measure outlined exclusively finished single tables. If it's not too much trouble take note of that the overarching record is entirely unexpected than "Join file", which may be relate get together of partner file planned keeps up sets of keywords of rows from 2 or a great deal of relations that match just if there should be an occurrence of a be a piece of [9][10]. These work races a two-way be a piece of question communicated in HiveQL as underneath: 


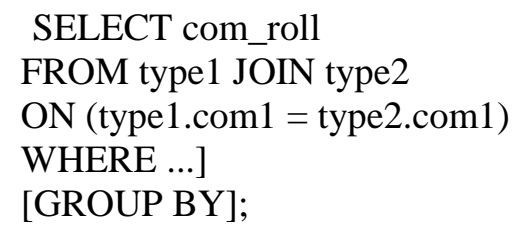

Wherever and group BY provisos are nonmandatory. Every one of our progressions are clear to the client and furthermore the linguistic structure of the inquiry stays in place. For delineation we tend to considered exclusively 2 tables, however our execution works easily for different tables comparably. The situation is, given 2 tables An, B with B have been ordered and an inquiry to hitch these 2 tables, execution be a piece of entire An and for each column in an exceedingly test the record on B. this is frequently gettable by recomposing the over inquiry into:

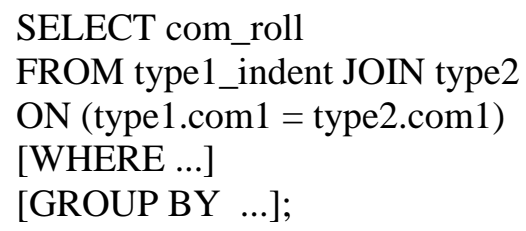

This change stream fits in with the normal change stream outline in Section II. Our usage utilizes the thoughts in HIVE-1694 and controls the inside learning structures inside the inquiry Processor; in any case, to manage it to strategy goes along with we tend to intercalary the expansion gave in Figure 3. Since the activity appeared inside the figure, the enhancer looks for a JoinOperator. On the off chance that this progression is precluded, the change is empowered for any inquiry. Our method is just stretched out to help multiway joins, by accomplishment this research, however since we have constraints over the pick section list we tend to chose to speak to our work for a two-way be a piece of. Inside the following stage we tend to get the Table Scan Operator those focuses to the table it should control. The list legitimacy check returns genuine if a table isn't apportioned off, or if it's parcels and that they aren't specified inside the wherever provision. Just in the event that its parcels and that they are said inside the wherever statement, it returns genuine if all the specified segments are covered by the record. When this progression the enhancer influences an endeavor to re-to compose the inquiry.

In the event that any of the conditions isn't met inside the stream portrayed in Figure 3, the technique closes in "Leave" that at that point infers that the execution result as was normal while not exploitation the list. It's key to state that, since there's no entrance to the base table, there's no entrance to all or any of its segments either. Rather, an arrangement of the traits (the ones that range unit filed) is offered once the re-compose. This restricts the inquiries which will be dealt with to exclusively questions referencing those particular sections. Our examinations and results region unit outlined next. 


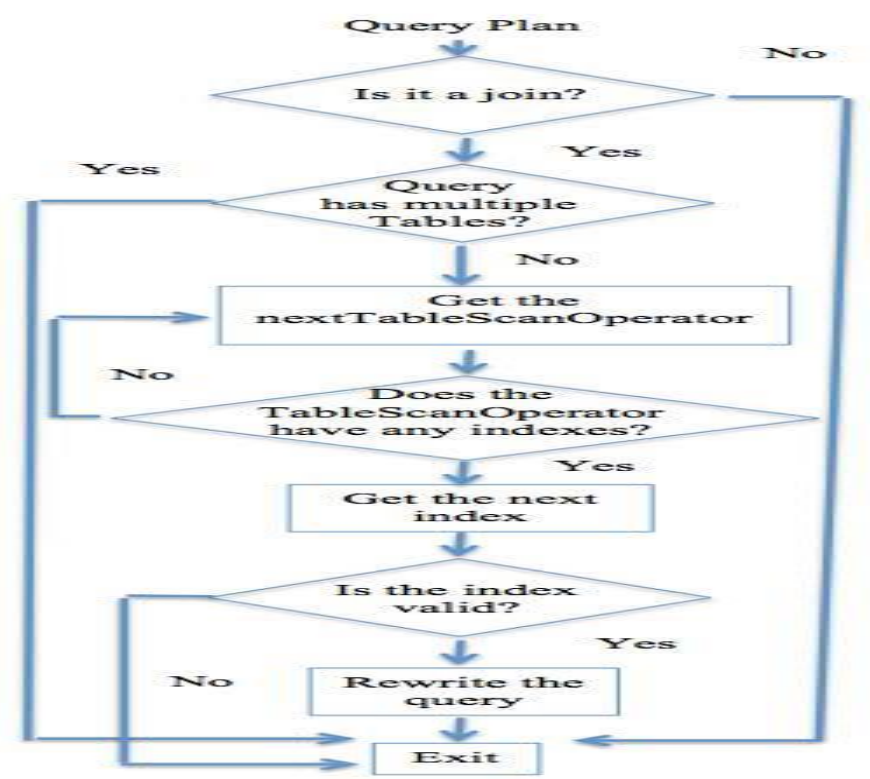

Figure 3. Flow of Optimization

\section{Experiments and Test RESULTS}

\section{Experiment 1:}

The present experiment incorporates execution of the four inquiry assortments, everyone is dead five times, on a multi-hub and a solitary hub Hadoop group exploitation five totally unique dataset sizes with line thing holding practically $5 / 6$ of the general information and scope of tuples beginning from in regards to $7 \times 106$ to $150 \times 106$. Figures four to seven delineate the normal inactive period for each information estimate. Inside the multi-hub setup, stirring from 1GB of learning to 20GB, by and large strides our record based approach beats the present one. The bigger the data region unit, the bigger the hole between the list less and record based methodologies moves toward becoming. Our record method is kind of twofold speedier than the file less approach by and large charts. Inside the single-hub setup, we tend to see indistinguishable conduct; for each learning size, our arranged strategy outflanks the conventional one and furthermore the bigger the data region unit, the bigger the hole between the record less and list approaches progresses toward becoming. The record procedure is kind of with respect to twofold speedier than the file less approach.

The best examination was the execution of our procedure, inside the remaining set of procedures or the experiments one, we tend to lead consistent check with totally unique questions, that are expansions of query1.Looking at Figures four to seven, the diagrams demonstrate comparative bends, exploitation that we tend to over that the four sorts of inquiries have almost steady conduct and that they neglected to bring about significantly totally extraordinary reaction times in neither methodologies. The most beyond a reasonable doubt won administrator on the whole the inquiries is that be a piece of. Neither wherever nor bunch BY, that wherever extra conditions extra to inquiries 2-4, starts a fresh out of the plastic new mapreduce work. The amount of mapreduce occupations everything considered the inquiries are satisfactory to one. Therefore, inside the rest of the trials we have a tendency to exclusively utilize question one. We conjointly examined the estimation of record creation as far as your chance and house to decide if or to not utilize list. 
Query1

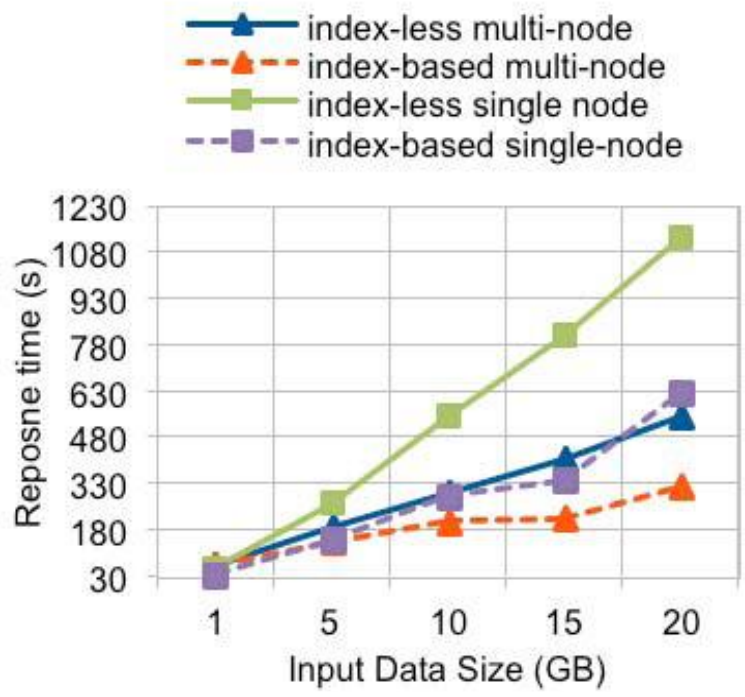

Figure 4. The Representation of Query 1 Response Time without index on Multi and Single-node Setups

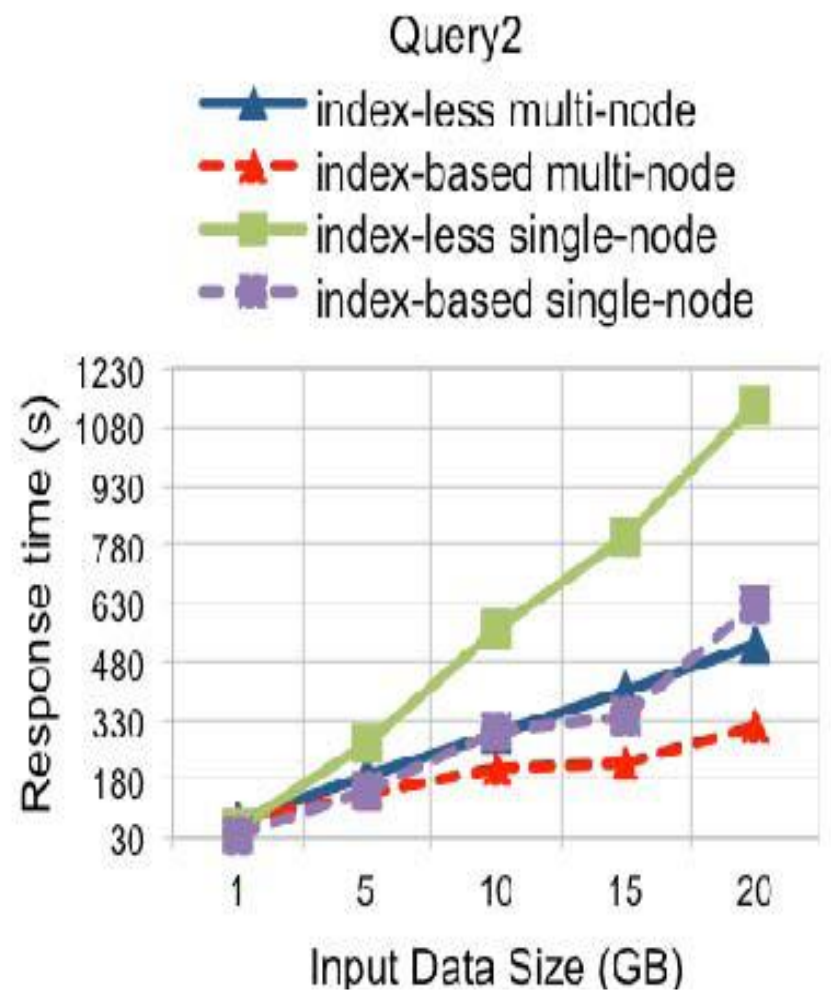

Figure 5. The Representation of Query 2 Response Times without Index on Multi and Single-node Setup 


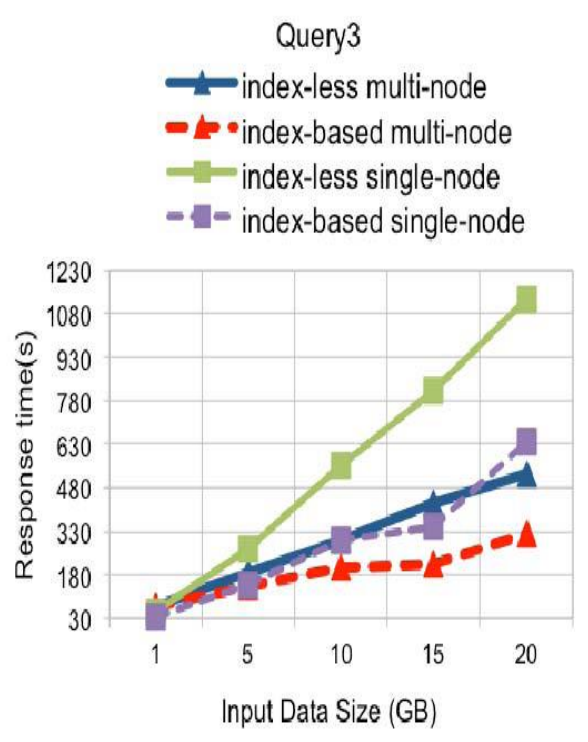

Figure 5. The Representation of Query 3 Response Times without Index on Multi and Single-node Setups

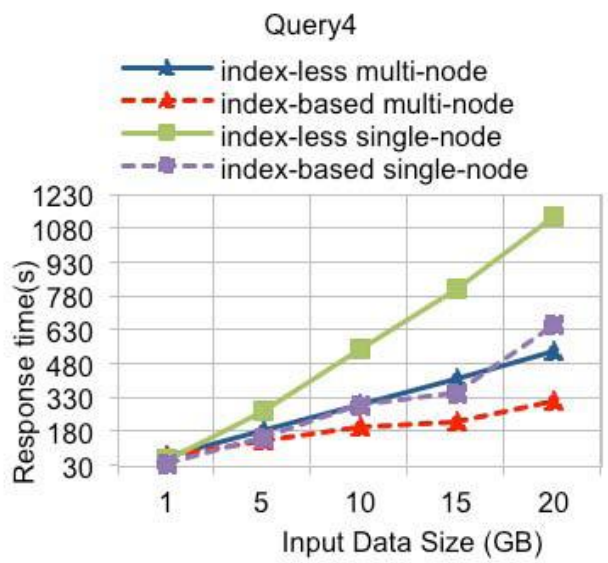

Figure 7. The Representation of Query 4 Response Times without Index on Multi and Single-node Setups

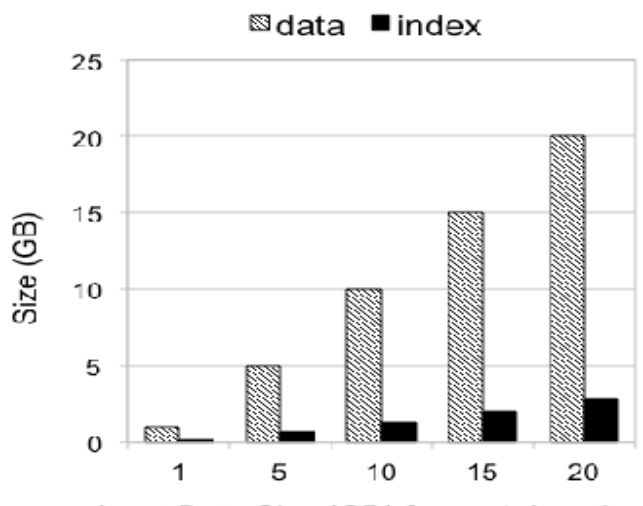

Input Data Size (GB) for each iteration

Figure 8. The Representation of the Difference between Size of the Index with the Size of the Data 


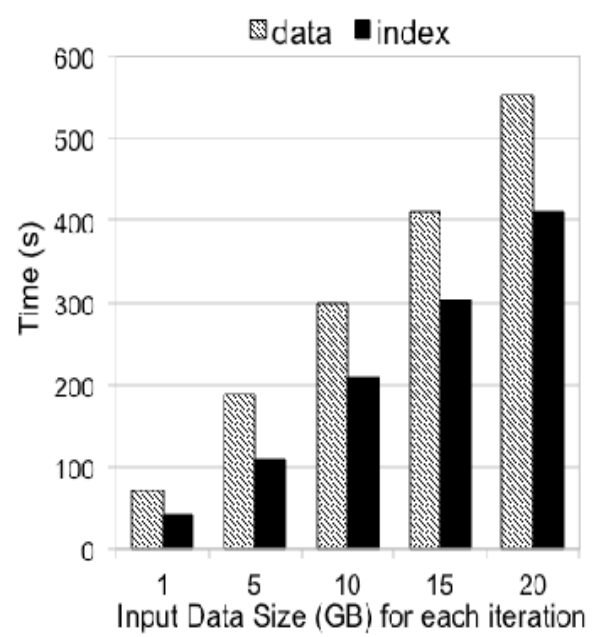

\section{Figure 9. The Representation of the Difference between Creation Time of the Index with the Response Time of the Query}

\section{Experiment 2:}

The second arrangement of tests we have a tendency to direct for execution measuring pondered entirely unexpected worth for the inquiry property proportions. For this reason, we have a tendency to utilized Query1 over the table's requests having a fixed size with fifteen $\times 105$ rows and conjointly table purpose of size beginning from zero.71 GB to ninety. 6 GB and with the measure of tuples beginning from six $\times 106$ to $7 \times 108$. in order to broaden the property, the street thing unmistakable be a piece of key or the yield size of the inquiry was unbroken at one, 500,000 though the information was multiplied whenever. Amid this test, we have a tendency to be intrigued to search out the reason at that our record based method functions distinguishably higher than the file less approach on our momentum multi-hub setup. Fig. ten demonstrates the charts for normal reaction times measured. As we tend to move from case one to eight amid this figure, the file less approach develops non-straightly, while the list based generally approach remains a considerable measure of or less steady at a middle of with respect to eighty seven seconds.

On the off chance that 7 , with $45 \mathrm{~GB}$ of data and zero.3\% as question property, the record based approach is A request of extent quicker than the file less approach. Future cycle, case 8 , with twofold inquiry property $(0.1 \%)$ and twofold learning size (90GB), our approach is twenty times snappier than the record less system. The exponential conduct of the file less diagram in Fig. 10, began at emphasis six with zero.7\% in light of the fact that the inquiry property. On the off chance that the bend keeps indistinguishable pattern, our list based approach will most likely be two requests of size speedier than the file less approach at $45 \mathrm{~TB}$ of data with unpleasantly particular $(0.0007 \%)$ questions. As demonstrated in Figure 12, the list estimate a tiny bit at a time drops from eighteen of the data size to $9 / 11$ over the eight cycles. The Hive list measure develops or contracts corresponding to the data size or appropriation. In Experiments two, the list diminishing rate is inferable from the data conveyance, as at each emphasis, the measure of unmistakable estimations of all characteristics, was unbroken indistinguishable while the measure of data was multiplied. In pertinence list development time, in Figure 12, we can see that, up to cycle five, file creation time is marginally however the execution of question one while not file, and surpasses the inquiry run-time accordingly. 


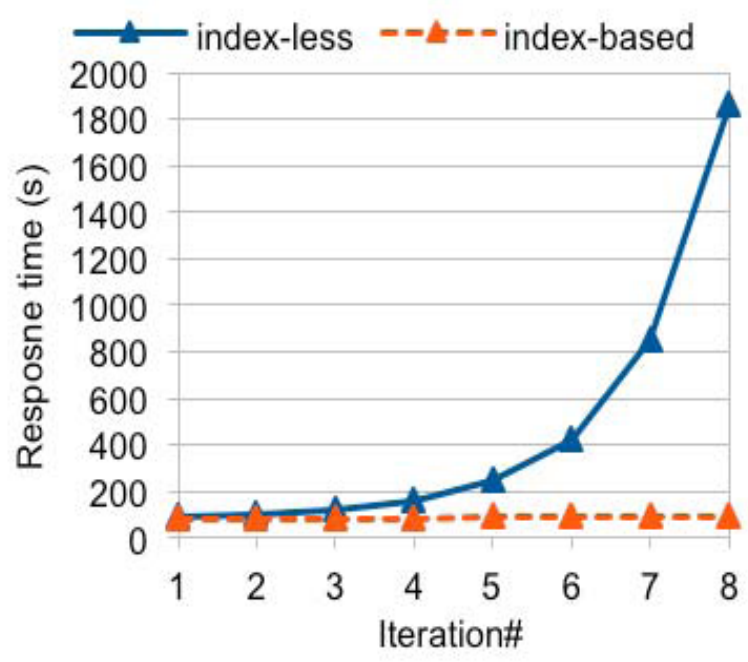

Figure 10. The Representation of the Query 1 Response Time without Index on Multi-node and Single-node Setup

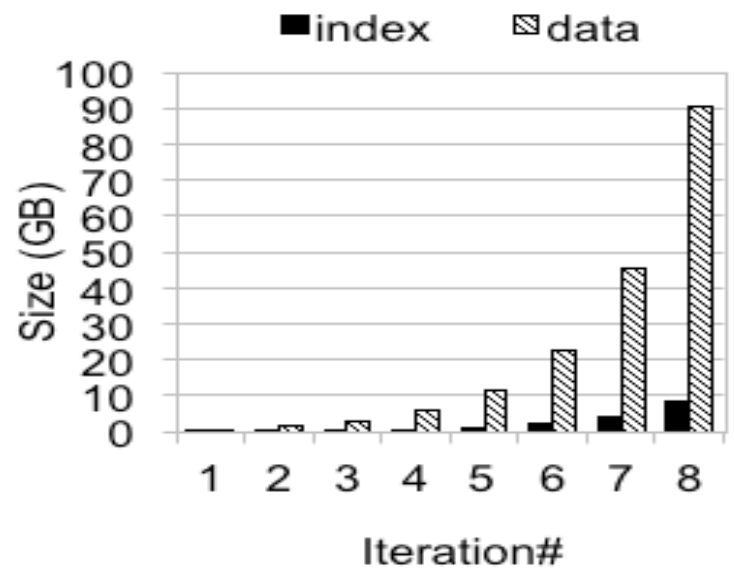

Figure 11. The Representation of the Difference between Size of the Index with the Size of the Data

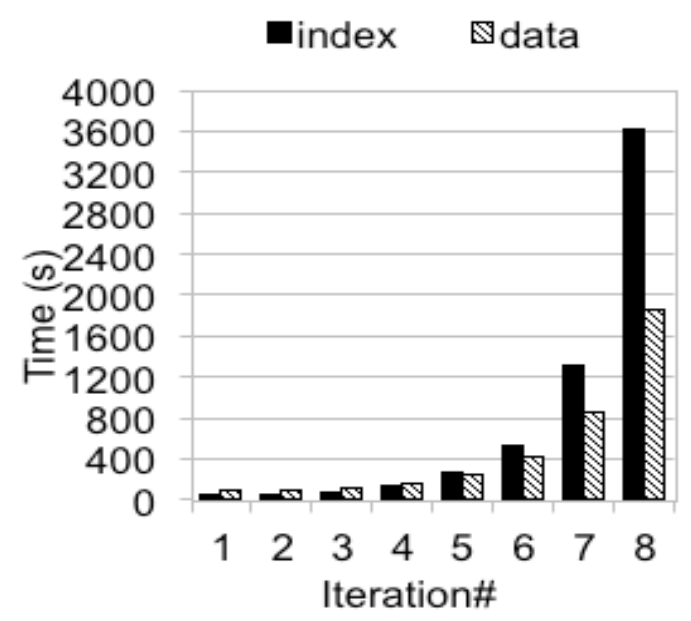

Figure 12. The Representation of the Difference between Creations of Index to the Response Time of Query 


\section{Conclusion}

Files are around for long-standing and furthermore the upside of exploitation them is reported. Notwithstanding, choosing once to utilize lists amid a situation needs escalated examination and exchange off between its cost and execution. Amid this investigation, we tend to utilize the present Hive arrangement structure to hustle just a bit be a piece of questions. From Experiments one, we tend to decided, when all is said in done, bigger the data region unit, bigger the execution pick up progresses toward becoming. Our approach developed directly on the whole cases appeared in Figures four to seven. In Experiment 2, we tend to gather the sizes of the datasets with developing property proportions. The aftereffects of those examinations demonstrated that our approach is exponentially faster than the present Hive approach. We found in Fig. 8, that the record estimate was about secured at exclusively V-day of the data measure in Experiment 1 and in Fig. 11, it took a middle of twelve-tone arrangement of the information in Experiment two. In spite of the fact that list estimate relies upon the data dispersion and furthermore the scope of properties for classification, our trials demonstrated the Hive file zone usage is modest. List creation time diagrams envisioned in Figures nine partner degreed twelve demonstrated the time required on building a file depended on the data conveyance, the a great deal of copied tuples came about amid a slower record creation strategy progressed toward becoming. In Figure 11, the most pessimistic scenario (cycle 8) record creation took about twofold the inquiry execution time. List development incorporates of perusing the aggregate information, arranging it, and wiping out the copies that might be a much extended strategy. Till the information present on the current base is not disturbed, any assortments of inquiries that have the benefit to use the list will utilize the record despite the list creation cost is quite recently acquired single time only. Hive list support cost is recognizably low, considering the uncommon updates and cluster mode information addition in light of the fact that the qualities of gigantic learning. In the event that new learning zone unit stacked into a substitution parcel of a base table, records are frequently made powerfully for that segment and unbroken one by one with none must be constrained to perform high-ticket refresh operations. Clearly, in Hive oversaw tables learning range unit examine twofold. Once to repeat it to the base table and once to make the record. The past are frequently wiped out if the record are regularly made inside the foundation while stacking learning into a table.

\section{References}

[1] P. Wang, "An efficient mapreduce algorithm for join in metrics", IJS, vol. 72, no. 3, (2016), pp. 11791200.

[2] Rasin et al., "A hybrid architecture for mapreduce and DBMS Technologies for Analytical Workloads", PVLDB, vol. 2, no. 1, (2010).

[3] K. Shvachko, "The Hadoop Distributed File System", IEEE Conference on MSST, NV, (2010), pp.1-10.

[4] S. Ghemawat, "MapReduce: Simplified Data Processing on Large Clusters", Magazine of Communications, ACM, vol. 51, no. 1, (2008), pp. 107-113.

[5] S. Agarwal, "Comparative study on Big Data Analytics and storing tools", (2016).

[6] Y. Wang, "Utilizing Index in the MapReduce Framework", "Twelth International Asia Pacific Web Conference, China, (2010), pp. 52-58.

[7] N. Jain and J. Liu, "Hive - A Petabyte Scale Data Warehouse Using Hadoop", IEEE International Conference on Information Engineering, (2010), pp. 996-1005.

[8] C. Zhang, "Optimizing the Theta Joins in Mapreduce environment", IJDTA (ISSN: 2005-4270), vol. 6, no. 1, (2013).

[9] P. Valduriez, "Join Indicies", ACM Transaction on Database Systems, vol. 12, no. 2, (1987), pp. 218264.

[10] A. Ross, "Quick joins utilizing join lists", The International Journal on Very Large Data Bases, vol. 8, no. 1, (1999), pp. 1-24.

[11] E. Omnecinski, "Inquiry Optimization utilizing segment insights in Hive", International Conference on Database Engineering and Applications, (2011), pp. 97-105. 
International Journal of Database Theory and Application Vol.10, No.9 (2017) 